\title{
Clinical significance of sHER2-ECD and calpain-10 expression in tumor tissues of patients with breast cancer
}

\author{
YAWEN DING ${ }^{1}$, MING MA $^{1}$, QINGJING LI ${ }^{1}$, SHEJUN GAO $^{1}$, \\ SAINAN $\mathrm{LI}^{2}$, JUNYING LIU ${ }^{3}$ and CUIZHI GENG ${ }^{2}$ \\ ${ }^{1}$ Clinical Laboratory; ${ }^{2}$ Breast Center; ${ }^{3}$ Department of Pathology, The Fourth Hospital of Hebei Medical University, \\ Shiljiazhuang, Hebei 050011, P.R. China
}

Received June 8, 2019; Accepted February 26, 2020

DOI: $10.3892 /$ or.2020.7564

\begin{abstract}
Human epidermal growth factor receptor 2(HER2) is composed of an extracellular domain (ECD), a lipophilic transmembrane region and an intracellular domain (ICD). The most commonly used method to determine the status of HER2 is immunohistochemistry. However, false-negative results are sometimes given, which causes some patients to lose the opportunity for anti-HER2 therapy. We found that calpain-10 may prohibit HER2-ECD into peripheral blood resulting in a HER2-negative result by the immunohistochemical method. We enrolled 289 patients into our experiment to assess the relationship between sHER2-ECD and calpain-10. The results showed that there was a positive correlation between sHER2-ECD and calpain-10. Moreover, we also investigated the prognostic values of sHER2-ECD and calpain-10 in breast cancer patients. According to the follow-up results, positive sHER2-ECD and tissue calpain-10 were indicative of a poor prognosis in breast cancer patients. Subsequently, we further validated the relationship between the two molecules in in vitro experiments. In the in vitro experiments, the level of HER2-ECD in the culture medium was increased or decreased with a decrease or increase in calpain-10 by transfection technology, showing an inverse association. The results indicated that sHER2-ECD and tissue calpain-10 levels were powerful factors to assess the status of HER2. In combination with tissue HER2 detection, the occurrence of false-negative HER2 was reduced, providing patients with additional treatment opportunities. In conclusion, sHER2-ECD and tissue calpain-10 may be used as new prognostic indices for breast cancer.
\end{abstract}

Correspondence to: Professor Cuizhi Geng, Breast Center, The Fourth Hospital of Hebei Medical University, 169 Tianshan Street, Shiljiazhuang, Hebei 050011, P.R. China

E-mail: cuizhigeng2019@hotmail.com

Key words: breast cancer, sHER2-ECD, HER2, calpain-10, prognosis

\section{Introduction}

As an independent prognostic factor, human epidermal growth factor receptor 2 (HER2) overexpression has been demonstrated to play a crucial role in overall survival (OS) and disease-free survival (DFS) of invasive breast cancer (BC) (1). It is particularly important to correctly evaluate the molecular state of HER2. HER2 is a $185-\mathrm{kDa}$ transmembrane receptor and a dimer with tyrosine kinase activity. It is composed of an extracellular domain (ECD) which can be combined with other members of the HER2 family, a lipophilic transmembrane region and an intracellular domain (ICD) with tyrosine kinase activity (2). Clinically, HER2 status is determined by immunohistochemistry. HER2 (3+) or HER2 $(2+) /$ in situ fluorescence hybridization (FISH) positive is defined as HER2 overex pression/positive, and HER2 (2+)/FISH negative or HER2 (0-1+) is defined as HER2-negative/low expression. HER2 positivity reflects a good prognosis for the application of anti-HER2 targeted therapy. However, not all HER2-positive patients are effectively treated with targeted therapy, while approximately $7 \%$ of patients with HER2 (0) can achieve good targeted therapeutic effects (3). Panis et al (3) found that $16 \%$ of HER2-negative and 30\% of HER2-positive BC patients are HER2-ICD positive, while free HER2-ECD is found in peripheral blood (4). The shedding of HER2-ECD has been linked to several proteolytic mechanisms that shed the ECD into the blood, and the remaining HER2-ICD fragments have the potential to induce effective cell signaling (5). The clinical anti-HER2 treatment is effective in some HER2-negative BC patients. In some patients with HER2-positive BC, ablation of ECD renders targeted therapy ineffective. Panis et al (3) compared the proteomics of serum HER2-positive and HER2-negative BC patients using high-throughput sequencing technology, and found that patients with HER2-negative BC and calpain-10 overexpression exhibit positive HER2-ICD. Therefore, we hypothesized that calpain-10 plays an important role in the shedding of ECD into the blood. In the present study, we aimed to explore the relationship among HER2, calpain-10 and serum HER2-ECD (sHER2-ECD). Moreover, we also investigated the role of these three indicators in the diagnosis and treatment management of BC patients. 


\section{Materials and methods}

Patient selection. A total of 289 BC patients without any tumor-related treatment and any other malignant diseases from April 2016 to October 2016 were enrolled at the Fourth Hospital of Hebei Medical University in the present study. The experimental protocols were approved by the Ethics Committee of the Fourth Hospital of Hebei Medical University, and informed consent was obtained from all participants. The diagnosis and treatment were standardized according to the National Comprehensive Cancer Network (NCCN) guidelines (https://www.ncen.org/), and the clinical and pathological characteristics of each patient were examined and recorded.

\section{Sample detection}

Detection of ER, PR, HER2 and calpain-10. The immunohistochemical status of the estrogen receptor (ER), progesterone receptor (PR), HER2 and calpain-10 was determined using the pathological tissues collected from the initial biopsy or surgery before the patient's first admission without any treatment. The tumor samples were first fixed with $4 \%$ neutral (phosphate buffer) formaldehyde fixative solution for $24 \mathrm{~h}$, and then paraffin-embedded tissue blocks were cut into $4-\mu \mathrm{m}$ sections. Subsequently, the expression levels of ER, PR, HER 2 and calpain-10 in BC tissues were examined by the S-P method. Immunohistochemical kits for ER, PR and HER2 were purchased from Ventana Medical Systems, Inc. Immunohistochemical kit for calpain-10 was obtained from Abcam. Primary antibodies against calpain-10 (dilution 1:1,000, product code ab28226; Abcam), ER (dilution 1:10; cat. no. 790-4325; Ventana Medical Systems, Inc.), PR (dilution 1:100; cat. no. 790-4296; Ventana Medical Systems, Inc.) and HER2 (dilution 1:100; cat. no. 790-4493; Ventana Medical Systems, Inc.) were supplied from available commercial sources. Known BC specimens were used as positive controls, and PBS was used as the negative control instead of the primary antibody. When the immunohistochemical result of HER2 was 2+, FISH detection was further performed. Formalin-fixed paraffin-embedded tissue was examined using the HER-2 DNA probe kit (Abbott) following the manufacturer's instructions. The ratio of LSI HER2/neu to CEP17 was determined via dividing the total number of LSI HER2/neu signals by the total number of CEP17 signals in the same 20 nuclei as previously described. If the ratio was $\geq 2$, HER2/neu gene amplification was observed.

Determination criteria of $E R, P R, H E R 2$ and calpain-10 outcomes. According to the above-mentioned immunohistochemical test results, the determination criteria for ER/PR results were set as follows: Brown-yellow staining of cancer cell nuclei $<10 \%$ was defined as negative expression of ER/PR, while $\geq 10 \%$ was defined as positive expression (6).

The determination criteria for HER2 results were set as follows: i) attention to the proportion of cancer cells with complete staining of the cell membrane and the staining intensity; ii) cytoplasmic staining was ignored; iii) the staining of intraductal carcinoma was ignored, and only the staining of infiltrating carcinoma was evaluated; iv) normal breast epithelium should not be stained. HercepTest scoring standard recommended by American Society of Clinical Oncology and
College of American Pathologists was applied (7). Results were scored from (-) to (3+). Briefly, no staining at all or less than $10 \%$ of the cell membrane staining was defined as (-); $\geq 10 \%$ of the cancer cells exhibiting weak and incomplete cell membrane staining was defined as (+); more than $10 \%$ of the cancer cells showing weak to medium intact cell membrane staining was defined as (2+); and more than $10 \%$ of the cancer cells showing strong, intact cell membrane staining was defined as (3+). In this test, $(-$ to +$)$ was considered as low expression, while (+++) was regarded as high expression. Formalin-fixed, paraffin-embedded tissues of HER2 (2+) patients underwent FISH test using the HER-2 DNA probe kit according to the manufacturer's instructions.

Both the staining intensity and the proportion of tumor cells was taken into consideration since the result of immunohistochemical staining was an average score. Each specimen was independently interpreted by two pathologists who were blinded to the patient information. A positive response was defined as the presence of a brown signal in the cytoplasm. For calpain-10, the staining index (0-12) was determined via multiplying the staining intensity score by the positive proportion score. The score of staining intensity was set as follows: Negative, 0 ; weak, 1; medium, 2; strong positive, 3 . The score of the proportion of positive cells was set as follows: $0,<5 \%$; $5-25 \% ; 2,26-50 \% ; 3,51-75 \% ; 4,>75 \%$. When the staining was not uniform, the score was defined as follows: Each component was independently scored, and the results were summarized. In the statistical analysis, a score of 0-7 was considered as low expression, while a score of 8-12 was considered as high expression.

sHER2-ECD detection. The sHER2-ECD detection was performed using the reagents approved by the FDA. Blood samples were drawn from patients who did not receive any radiotherapy, chemotherapy and surgery before any treatment. Subsequently, $5 \mathrm{ml}$ peripheral venous blood was centrifuged at $1,912 \mathrm{xg}$ for $10 \mathrm{~min}$ at room temperature, and serum was collected, followed by detection of serum HER2-ECD using the HER2-ECD detection kit. Test results $>15 \mathrm{ng} / \mathrm{ml}$ were considered positive.

Follow-up. All the enrolled patients were closely followed up until January 2019 with the disease progression (including recurrence, metastasis or death) as the follow-up endpoint, and the follow-up period ranged from 23 to 30 months. The correlations between HER2-ECD or calpain-10 and OS, DFS and the cumulative incidence rate (CIR) were analyzed. DFS was defined as the time from randomization to the first event of either disease recurrence or death due to any cause. OS was defined as the time from the date of randomization to the date of death due to any cause. CIR was defined as the frequency of recurrence after a specific observation period (typically more than 1 year) in patients with complete remission.

In vitro studies. Five commonly used BC cell lines with different HER 2 expression status were selected, including MCF-7, MDA-MB-231, BT-549, MDA-MB-453 and SKBR3. The expression levels of calpain-10 and HER2 at the mRNA and protein levels in these cell lines were determined. 
$R T$ - $q P C R$. Human BC cell lines were maintained in DMEM medium (Gibco; Thermo Fisher Scientific, Inc.) supplemented with $10 \%$ fetal calf serum, $100 \mathrm{U} / \mathrm{ml}$ penicillin and $100 \mu \mathrm{g} / \mathrm{ml}$ streptomycin at $37^{\circ} \mathrm{C}$ in a humidified atmosphere containing $5 \% \mathrm{CO}^{2}$. Two HER2- overexpressing cell lines, BT-549 and SKBR3, were transfected with the CAPN10-human, 4 unique 29mer shRNA constructs in lentiviral GFP vector (OriGene Technologies) to block calpain-10 activity. Subsequently, the CAPN10 pENTER vector was transfected into BT-549 and SKBR3 cell lines to overexpress CAPN10. Treatment was performed under specific conditions. Forty hours after transfection, the subsequent procedures such as RNA and protein extraction were carried out.

The expression levels of calpain-10 and HER 2 at the mRNA level were determined by RT-qPCR. Total RNA was extracted by TRIzol reagent. Purified RNA was reversely transcribed into cDNA using a reverse transcription kit, and synthesized cDNA was used as a template for RT-qPCR using Platinum SYBR SuperMix on an ABI 7500 system (Promega). The primers were designed by iGeneBio Inc. as follows: Calpain-10 forward, 5'-GGGAGTTCCATGCCTTCATT-3' and reverse, 5'-TACCTGGCTCCACCCTT-3'; GAPDH forward, 5'-GTC AACGGATTTGGTCGTATTG-3' and reverse, 5'-TGGAAG ATGGTGATGGGATTT-3'; HER2 forward, 5'-TATGCAGGG CTGACGTAGTGC3' and reverse, 5'-AATGTGTGCCAC GAAACTGCT-3'. Briefly, amplifications were carried out with 40 cycles at a melting temperature of $95^{\circ} \mathrm{C}$ for $15 \mathrm{sec}$, an annealing temperature of $60^{\circ} \mathrm{C}$ for $30 \mathrm{sec}$, and an extension temperature of $72^{\circ} \mathrm{C}$ for $30 \mathrm{sec}$. GAPDH was used as the housekeeping gene. The relative expression of calpain-10 and HER2 was calculated using the $2^{-\Delta \Delta \mathrm{Cq}}$ method (8).

Western blot analysis. The expression of calpain-10 and HER2 at the protein level in five BC cell lines and transfected BT-549 and SKBR3 cells was determined by western blot analysis. Briefly, the cells were lysed in cold lysis buffer consisting of $50 \mathrm{mM}$ Tris (pH 7.5), $5 \mathrm{mM}$ EDTA, $10 \mathrm{mM}$ EGTA, $50 \mathrm{mM}$ $\mathrm{NaF}, 20 \mathrm{mM} \beta$-glycerophosphate, $250 \mathrm{mM} \mathrm{NaCl}, 2 \% \mathrm{NP}-40$ and protease inhibitors and incubated on ice for $30 \mathrm{~min}$. The cell lysates were centrifuged at $12,000 \mathrm{x}$ g for $15 \mathrm{~min}$ at $4^{\circ} \mathrm{C}$, and the supernatants were collected. BCA assay (Pierce; Thermo Fisher Scientific, Inc.) was used to evaluate the protein content. The protein was separated on 10\% SDS-PAGE gel and transferred to PVDF membranes (Pierce; Thermo Fisher Scientific, Inc.). The membranes were incubated in PBS containing $5 \%$ bovine serum albumin for $2 \mathrm{~h}$ at room temperature. Then the primary antibodies with different dilutions were cultured overnight at $4^{\circ} \mathrm{C}$. The antibodies were against HER2 (dilution 1:100, product code ab134182; Abcam), calpain-10 (dilution 1:1,000; product code ab28226; Abcam) and $\beta$-actin Ab (dilution 1:5,000; cat. no. 66009-1-Ig; Proteintec). Then culture was carried out with horseradish peroxidase-conjugated anti-rabbit secondary antibody for $2 \mathrm{~h}$ at room temperature. Each experiment was performed in triplicate. The level of HER2-ECD and calpain-10 in each sample was calculated as the ratio of the intensity of protein to that of $\beta$-actin, using Odyssey v3.0 software (LI-COR Biosciences).

Statistical analysis. For the clinical investigation, Chi-square test was used for comparison between groups. Correlation analysis was conducted using Pearson's correlation test, and survival curve analysis was performed using Log-rank test. For the in vitro experiments, t-test was used for comparison between the two groups, including Student's t-test and paired t-test. Bonferroni's test and one-way ANOVA were used for multiple comparisons. P-value $<0.05$ was considered as indicative of statistical significance.

\section{Results}

\section{Clinical investigation}

Basic data of the enrolled BC patients. All 289 patients with primary $\mathrm{BC}$ were females, and their median age was 57 years, ranging from 26 to 80 . There were 159 premenopausal women and 130 postmenopausal women. According to the TNM staging of the American Joint Committee on Cancer (AJCC) version 7 (9), there were 75 patients with stage I, 160 patients with stage II, 42 patients with stage III and 12 patients with stage IV. Moreover, 32 patients received neoadjuvant chemotherapy, and 4 patients of stage IV underwent surgery after chemotherapy. Among the 289 patients, 158 had no lymph node metastasis, and no angioma thrombus was found in 227 patients. Patients with pathological grade 1, 2 and 3 were 29, 166 and 94, respectively. There were 80 and 110 patients negative for ER and PR, respectively. HER2-ECD in peripheral blood was detected in 289 patients at first admission. According to the instructions of the reagents, the cut-off value was $15 \mathrm{ng} / \mathrm{ml}$. The results showed that 224 patients were negative and 65 patients were positive. Patients were divided into two groups according to the sHER2-ECD levels. Table I summarizes the detailed relationship between the HER2-ECD level and various clinicopathological factors. We collected the peripheral blood of 20 healthy adult women and tested sHER2-ECD. The detection value ranged from 4.6-11.8 ng/ml, with an average value of $7.6 \mathrm{mg} / \mathrm{ml}$. The value of HER2-ECD in healthy adult women were all negative.

There were no statistically significant differences in menstrual status, ER and PR levels between the sHER2-ECD-positive and sHER2-ECD-negative groups. According to TNM staging, the patients were divided into stage I, stage II, stage III and stage IV. Moreover, we found that in the patients with higher staging, the proportion of sHER2-ECD-positive patients was higher and the proportion of sHER2-ECD-negative patients was lower. There were 65 patients in the sHER2-ECD-positive group. Among these patients, lymph node metastasis was found in 47 patients (72.31\%), and only 18 patients $(27.69 \%)$ showed no lymph node metastasis. In the sHER2-ECD-negative group, 84 (37.5\%) had lymph node involvement, while the majority of them (140, $62.5 \%$ ) had no lymph node involvement. Angioma emboli were present in $40 \%(26 / 65)$ of the sHER2-ECD-positive patients, while this proportion was only $19.15 \%(36 / 224)$ in the sHER2-ECD-negative group. The higher the pathological grade, the higher the proportion of sHER2-ECD-positive patients and the lower the proportion of sHER2-ECD-negative patients. Tissue specimens obtained by coarse needle aspiration both before neoadjuvant chemotherapy and surgery were collected from the Department of Pathology, The Fourth Hospital of Hebei Medical University and the histological response to chemotherapy in the breast was assessed by two 
Table I. Association between serum sHER2-ECD levels and clinicopathological factors of the BC patients ( $\mathrm{n}=289)$.

\begin{tabular}{|c|c|c|c|c|}
\hline \multirow[b]{2}{*}{ Characteristics } & \multicolumn{2}{|c|}{ sHER2-ECD } & \multirow[b]{2}{*}{$\chi^{2}$} & \multirow[b]{2}{*}{ P-value } \\
\hline & $\begin{array}{c}<15 \mathrm{ng} / \mathrm{ml} \\
\mathrm{n}(\%)\end{array}$ & $\begin{array}{c}\geq 15 \mathrm{ng} / \mathrm{ml} \\
\mathrm{n}(\%)\end{array}$ & & \\
\hline \multicolumn{5}{|l|}{ Menopausal status } \\
\hline Premenopausal & $120(75.47)$ & $39(24.53)$ & 0.841 & 0.359 \\
\hline Postmenopausal & $104(80.00)$ & $26(20.00)$ & & \\
\hline \multicolumn{5}{|l|}{ Stage } \\
\hline I & $72(96.00)$ & $3(4.00)$ & 36.848 & $<0.001$ \\
\hline II & $121(75.63)$ & $39(24.37)$ & & \\
\hline III & $28(66.67)$ & $14(33.33)$ & & \\
\hline IV & $3(25.00)$ & $9(75.00)$ & & \\
\hline \multicolumn{5}{|l|}{ Nodal invasion } \\
\hline No & $140(88.61)$ & $18(11.39)$ & 24.631 & $<0.001$ \\
\hline Yes & $84(64.12)$ & $47(35.88)$ & & \\
\hline \multicolumn{5}{|c|}{ Carcinoma cell embolus } \\
\hline No & $188(82.82)$ & $39(17.18)$ & 17.119 & $<0.001$ \\
\hline Yes & $36(58.06)$ & $26(41.94)$ & & \\
\hline \multicolumn{5}{|l|}{ Grade } \\
\hline G1 & $27(93.10)$ & $2(6.90)$ & 8.198 & 0.017 \\
\hline $\mathrm{G} 2$ & $132(79.52)$ & $34(20.48)$ & & \\
\hline G3 & $65(69.15)$ & $29(30.85)$ & & \\
\hline \multicolumn{5}{|l|}{ MP grade } \\
\hline $1-4$ & $17(58.62)$ & $12(41.38)$ & 0.088 & 0.044 \\
\hline 5 & $1(14.29)$ & $6(85.71)$ & & \\
\hline \multicolumn{5}{|c|}{ Receptor status (cut-off $\geq 10 \%$ ) } \\
\hline \multicolumn{5}{|l|}{ ER } \\
\hline- & $58(72.5)$ & $22(27.5)$ & 1.592 & 0.207 \\
\hline+ & $166(79.43)$ & $43(20.57)$ & & \\
\hline \multicolumn{5}{|l|}{ PR } \\
\hline- & $85(77.27)$ & $25(22.73)$ & 0.006 & 0.940 \\
\hline+ & $139(77.65)$ & $40(22.35)$ & & \\
\hline \multicolumn{5}{|c|}{ HER2 status (IHC/FISH) } \\
\hline- & $107(87.70)$ & $15(12.30)$ & 12.591 & $<0.001$ \\
\hline+ & $117(70.06)$ & $50(29.94)$ & & \\
\hline
\end{tabular}

sHER2-ECD, serum human epidermal growth factor receptor 2-extracellular domain; ER, estrogen receptor; PR, progesterone receptor; MP, Miller and Payne.

senior pathologists using the Miller and Payne grading system: MP1, no change or some alterations to individual malignant cells but no reduction in overall cellularity; MP2, a minor loss of tumor cells but overall cellularity was still high (up to $30 \%$ loss); MP3, an estimated reduction between 30 and 90\% in tumor cells; MP4, a marked disappearance of tumor cells that only small clusters or widely dispersed individual cells remained (more than 90\% loss of tumor cells); and MP5, no identifiable malignant cells in sections from the site of the tumor (only vascular fibroelastotic stroma remained often containing macrophages). However, ductal carcinoma in situ may be present (10). In general, we defined MP5 as
pCR (11). Patients of grade MP1-4 had worse prognosis than those of grade MP5 (12). A total of 36 patients received neoadjuvant chemotherapy. For patients undergoing neoadjuvant chemotherapy, $33.33 \%(6 / 18)$ of the sHER2-ECD-positive patients reached grade MP5, while only one patient in the sHER2-ECD-negative group reached grade MP5, accounting for $5.56 \%(1 / 18)$, and the statistical difference between the two groups was significant $(\mathrm{P}<0.05)$. According to the immunohistochemical and/or FISH results, we found that the proportion of sHER2-ECD-positive patients in the sHER2-positive group $(29.94 \%, 50 / 167)$ was significantly higher than that in the sHER2-negative group $(12.30 \%, 15 / 122)(\mathrm{P}<0.05)$. 
Table II. Relationship between HER2 tissue status and calpain-10 tissue status.

\begin{tabular}{lccccr}
\hline & \multicolumn{2}{c}{ Calpain-10 tissue status } & & & \\
\cline { 2 - 4 } & $\begin{array}{c}\text { Low expression } \\
\mathrm{n}(\%)\end{array}$ & $\begin{array}{c}\text { High expression } \\
\mathrm{n}(\%)\end{array}$ & $\chi^{2}$ & P-value & R-value \\
\hline HER2 tissue status & & & & & 0.123 \\
- & $75(61.48)$ & $47(38.52)$ & 4.350 & 0.037 & \\
+ & $82(49.10)$ & $85(50.90)$ & & & 0.123 \\
\hline
\end{tabular}

HER2, human epidermal growth factor receptor 2.
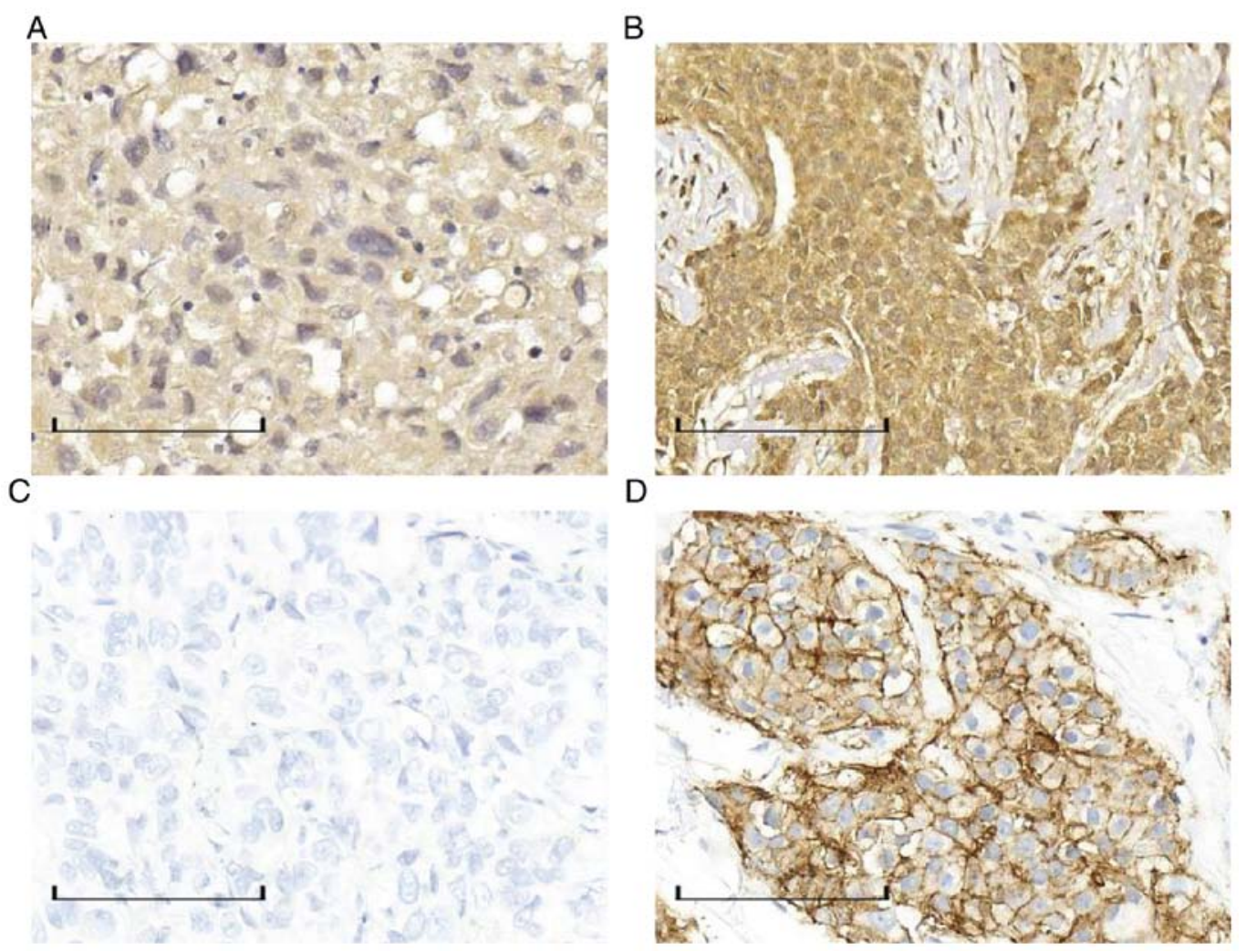

Figure 1. (A) Low expression of calpain-10 in breast cancer tissue. (B) Overexpression of calpain-10 in breast cancer tissue. (C) Negative expression of HER2 in breast cancer tissue. (D) Positive expression of HER2 in breast cancer tissue. magnification, x200; scale bar, $100 \mu \mathrm{m}$. HER2, human epidermal growth factor receptor 2 .

Relationship between HER2 and calpain-10. According to the above-mentioned scoring criteria, among the 289 enrolled patients, 54.33\% (157/289) had low expression of calpain-10 (Fig. 1A), and $45.67 \%(132 / 289)$ had overexpression of calpain-10 (Fig. 1B). The histological status of HER2 was significantly different between the negative group (Fig. 1C) and the positive group (Fig. 1D) $(\mathrm{P}=0.037)$, and there was a positive correlation between calpain-10 and HER2 $(\mathrm{R}=0.123)$ (Table II).

Relationship between SHER2-ECD and calpain-10. Most sHER2-ECD-negative patients showed low expression of calpain-10, while those with positive sHER2-ECD exhibited overexpression of calpain-10. Table III reveals that there was a positive correlation between sHER2-ECD and calpain-10 $(\mathrm{r}=0.439)$.

Follow-up. The DFS curve showed that patients in the sHER2-ECD-negative group had enhanced DFS than those in the sHER2-ECD-positive group $(\mathrm{P}<0.0001)$ (Fig. 2A). This finding suggests that positive sHER2-ECD is more likely to lead to enhanced disease progression (including recurrence, metastasis or death). These results demonstrated the prognostic value of sHER2-ECD.

Three patients died in the sHER2-ECD-negative group, 5 patients died in the positive group, and there was no significant difference with such a number of deaths and small number of cases. However, the survival time of the cases that 
Table III. Relationship between serum HER2-ECD status and calpain-10 tissue status.

Calpain-10 tissue status

\begin{tabular}{lccc} 
sHER2-ECD & $\begin{array}{c}\text { Low expression } \\
\text { n }(\%)\end{array}$ & $\begin{array}{c}\text { High expression } \\
\text { n }(\%)\end{array}$ & P-value \\
\hline$<15 \mathrm{ng} / \mathrm{ml}$ & $145(64.73)$ & $79(35.27)$ & $<0.001$ \\
$\geq 15 \mathrm{ng} / \mathrm{ml}$ & $8(12.31)$ & $57(87.69)$ & \\
\hline
\end{tabular}

sHER2-ECD, serum human epidermal growth factor receptor 2-extracellular domain.

Table IV. Molecular characteristics of the five cell lines.

\begin{tabular}{lccccc}
\hline Molecules & BT-549 & SKBR3 & MDA-MB-231 & MCF-7 & MDA-MB-453 \\
\hline HER2-ECD & 18.3 & 24.8 & 12.3 & 7.7 & - \\
HER2 & + & + & - & - & - \\
Calpain-10 & + & + & - & - \\
\hline
\end{tabular}

HER2-ECD, human epidermal growth factor receptor 2-extracellular domain.

A

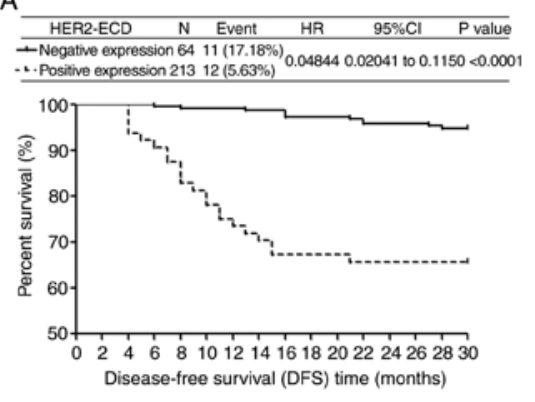

$\mathrm{C}$
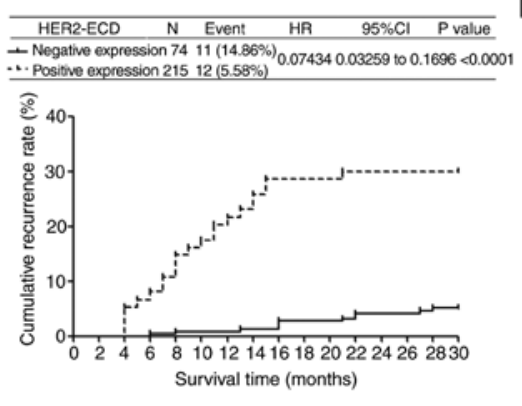

E

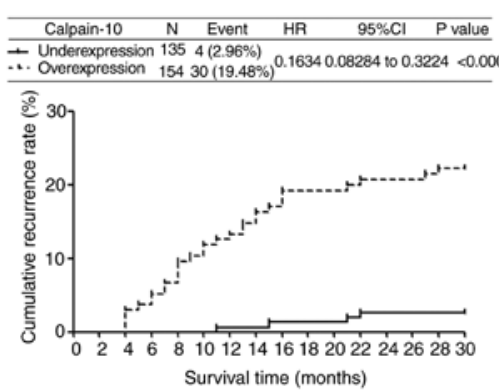

B

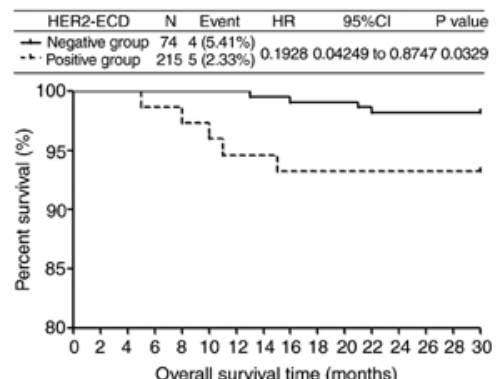

$\mathrm{D}$
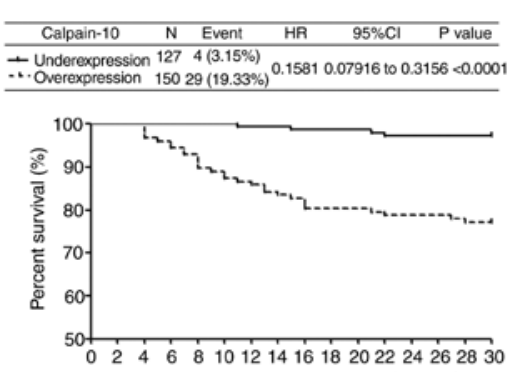

Disease-free survival (DFS) time (months)

$\mathrm{F}$

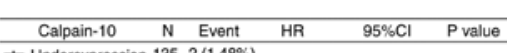

$\begin{array}{lll}- \text { Underexpression } 1352(1.48 \%) & 0.27920 .07527 \text { to } 1.0350 .0564\end{array}$
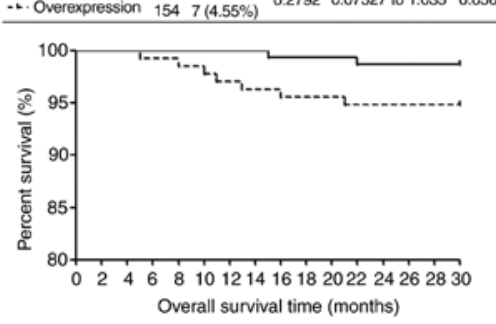

Figure 2. (A) DFS curve illustrates that patients in the HER2-ECD-negative group had longer DFS than those in the HER2-ECD-positive group. (B) A statistical difference in OS was found between the HER2-ECD-negative group and the HER2-ECD-positive group. (C) CRR in the HER2-ECD-positive group was significantly higher than that in the HER2-ECD-negative group. (D) DFS curve of calpain-10 was similar to that of the HER2-ECD. (E) CRR curve of calpain-10 was similar to that of HER2-ECD. (F) There was no significant difference in OS between the low-expression group and the overexpression group. DFS, disease-free survival; HER2-ECD, human epidermal growth factor receptor 2-extracellular domain; OS, overall survival; CRR, cumulative recurrence rate; $\mathrm{HR}$, hazard ratio; $\mathrm{CI}$, confidence interval. 

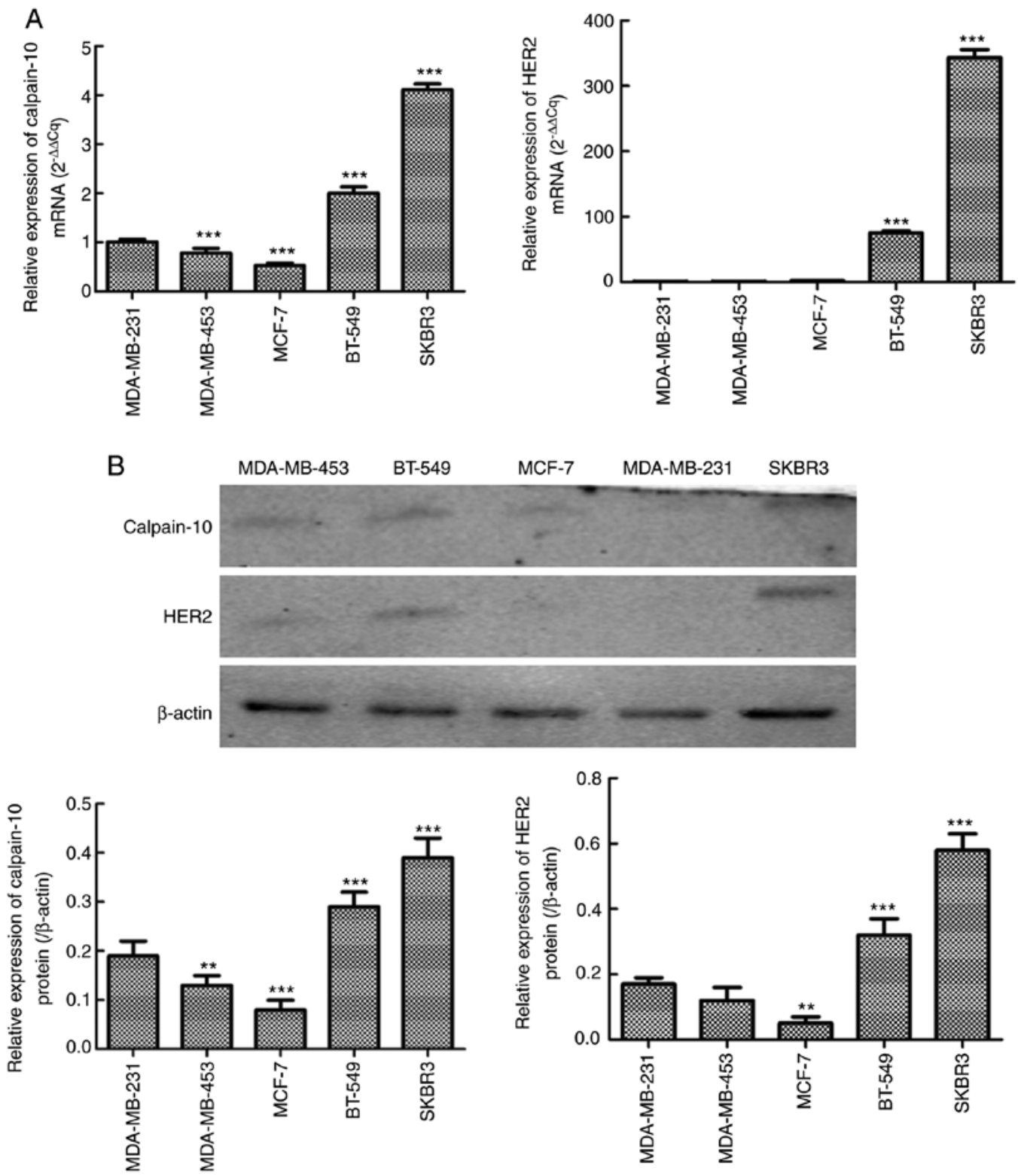

Figure 3. Expression of calpain-10 and HER2 at the mRNA and protein levels in MDA-MB-231, BT-549, SKBR3, MCF-7 and MDA-MB-453 cell lines. (A) Expression of calpain-10 and HER2 at the mRNA level in MDA-MB-231, BT-549, SKBR3, MCF-7 and MDA-MB-453 cell lines as detected by RT-qPCR. (B) Expression of calpain-10 and HER2 at the protein level in MDA-MB-231, BT-549, SKBR3, MCF-7 and MDA-MB-453 cell lines as detected by western blot analysis. ${ }^{* *} \mathrm{P}<0.01$ and ${ }^{* * *} \mathrm{P}<0.001$. HER2, human epidermal growth factor receptor 2.

succumbed to the disease in the positive group was shorter. Therefore, there was a statistical difference in OS between the two groups ( $\mathrm{P}=0.0329$; Fig. 2B).

The cumulative recurrence rate (CRR) in the sHER2-ECD-positive group was significantly higher than that in the sHER2-ECD-negative group $(\mathrm{P}<0.0001)$ (Fig. 2C), indicating that the sHER2-ECD-positive patients were more prone to disease progression and the prognostic value of sHER2-ECD.

The DFS and CRR curves of calpain-10 were similar to those of sHER2-ECD (Fig. 2D and E), indicating the prognostic value of calpain-10 and the positive correlation between calpain-10 and sHER2-ECD. This finding suggests that calpain-10 shears ECD and releases it into the blood. However, there was no significant difference in OS between the low-expression group and the overexpression group, which may have been attributed to the short follow-up time (Fig. 2F).

\section{In vitro experiments}

Expression of calpain-10 and HER2 at the $\mathrm{mRNA}$ and protein levels in different cell lines. Expression of calpain-10 and HER2 at the mRNA level in MDA-MB-231, BT-549, SKBR3, MCF-7 and MDA-MB-453 cell lines were detected by RT-qPCR. The results showed that the expression of calpain-10 and HER2 in BT-549 and SKBR3 cell lines were significantly higher compared with the other three cell lines (Fig. 3A). Western blot analysis showed the same results (Fig. 3B). We tested the expression of HER2-ECD in the culture medium of the five cell lines (Table IV). The average concentrations of HER2-ECD in BT-549 and SKBR3 cell lines were 18.3 and $24.8 \mathrm{ng} / \mathrm{ml}$, respectively, while the average concentrations in MDA-MB-231, MCF-7 and MDA-MB-453 cell lines were $12.3,7.7$ and $4.6 \mathrm{ng} / \mathrm{ml}$, respectively, which were all less than $15 \mathrm{ng} / \mathrm{ml}$. 

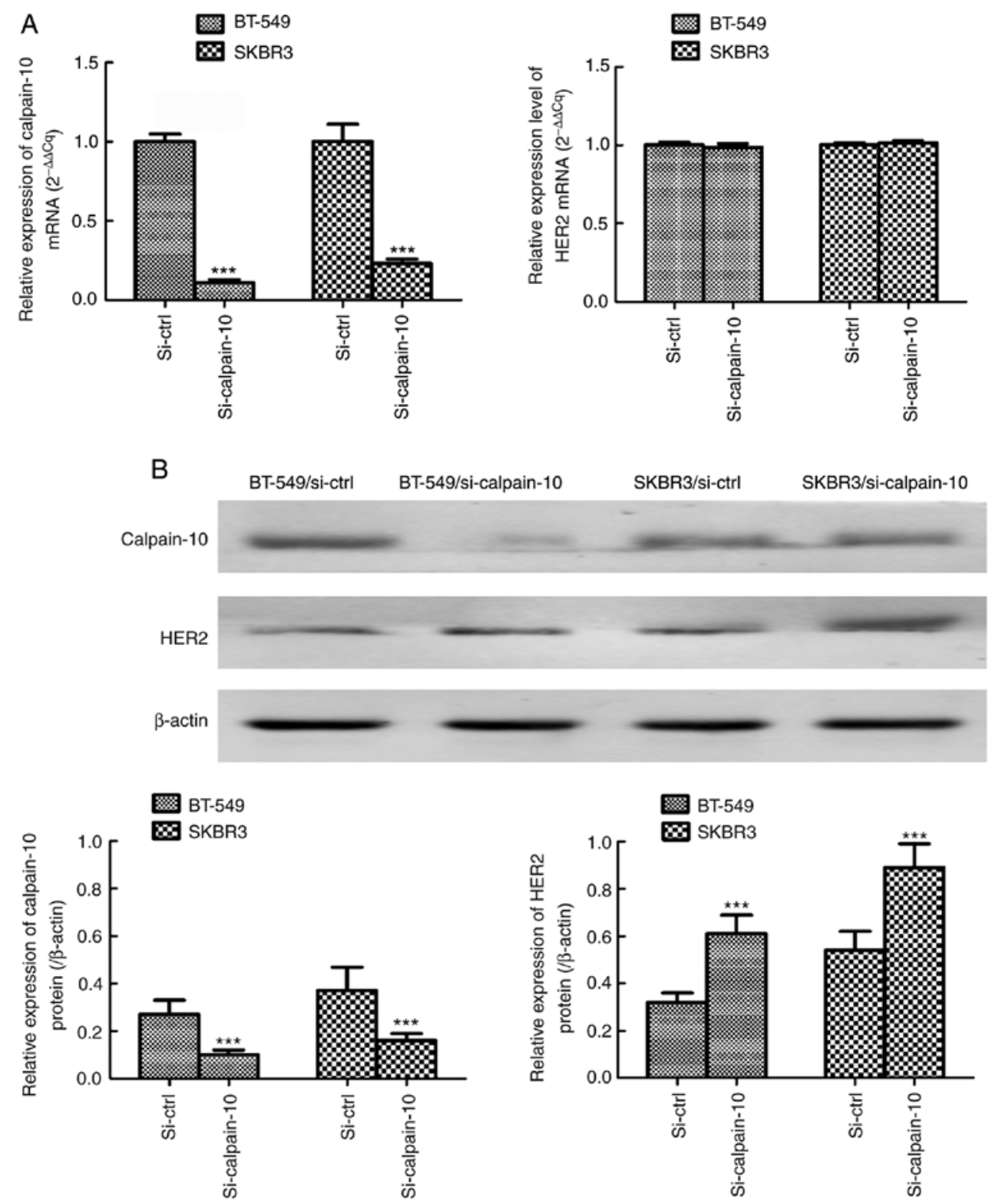

Figure 4. Relative expression of calpain-10 and HER2 in SKBR3 and BT-549 after transfection (downregulation of calpain-10) at the mRNA and protein levels. (A) Relative expression of calpain-10 and HER2 in SKBR3 and BT-549 after transfection (downregulation of calpain-10; Si-calpain-10) at the mRNA level. (B) Relative expression of calpain-10 and HER2 in SKBR3 and BT-549 after transfection (downregulation of calpain-10) at the protein level. ${ }^{* * *}$ P $<0.001$, compared with the Si-ctrl group. HER2, human epidermal growth factor receptor 2.

Next, we designed the following experiments for two cell lines (BT-549 and SKBR3) with high expression of calpain-10 and HER2. We transfected BT-549 and SKBR3 cells with CAPN10-human, 4 unique 29mer shRNA constructs in lentiviral GFP vector (si-calpain-10) that was able to downregulate calpain-10. We found that the expression of calpain-10 at the mRNA (Fig. 4A) and protein (Fig. 4B) levels in these two cell lines was significantly lower compared with the non-transfected group (si-ctrl). The expression level of HER2-ECD in the medium was also altered from positive to negative (Table V). Meanwhile, the expression of HER2 at the protein (Fig. 4B) level in the two strains was increased after transfection, while the expression of HER2 at the mRNA (Fig. 4A) level exhibited no significant change.

Subsequently, CAPN10 pENTER vector capable of overexpressing calpain-10 was transfected into the BT-549 and SKBR3 cells, in which the expression of calpain-10 at the mRNA (Fig. 5A) and protein (Fig. 5B) levels was higher than that of the non-transfected group (EV). The concentration of HER2-ECD in the BT-549 and SKBR3 cell culture media was increased obviously (Table VI). After simultaneous transfection, the expression of HER2 at the protein (Fig. 5B) level in the two cell lines was lower than that before transfection, while expression of HER2 at the mRNA (Fig. 5A) exhibited no significant change.

\section{Discussion}

Recently, more and more attention has been paid to the role of serum human epidermal growth factor receptor 2-extracellular domain (sHER2-ECD) in the evaluation of prognosis and treatment management of breast cancer (BC) patients. Among the factors related to the prognosis of $\mathrm{BC}$, advanced clinical 

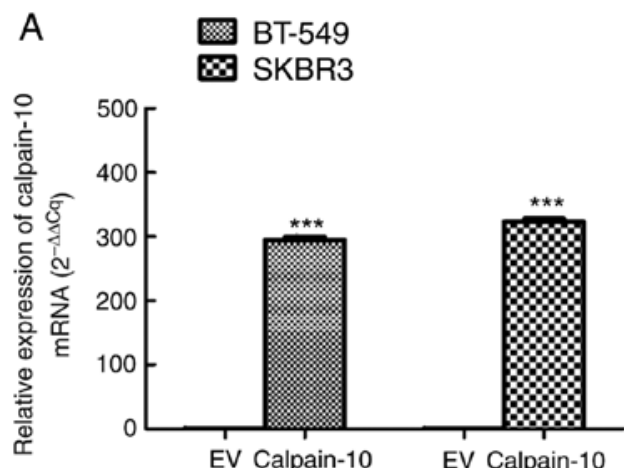

B

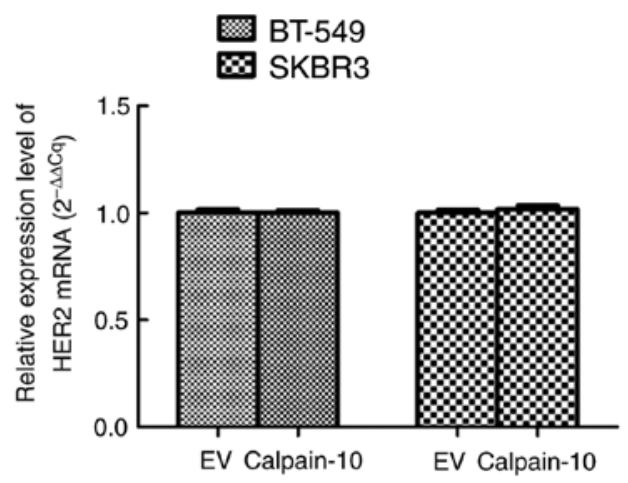

SKBR3/EV SKBR3/Calpain-10
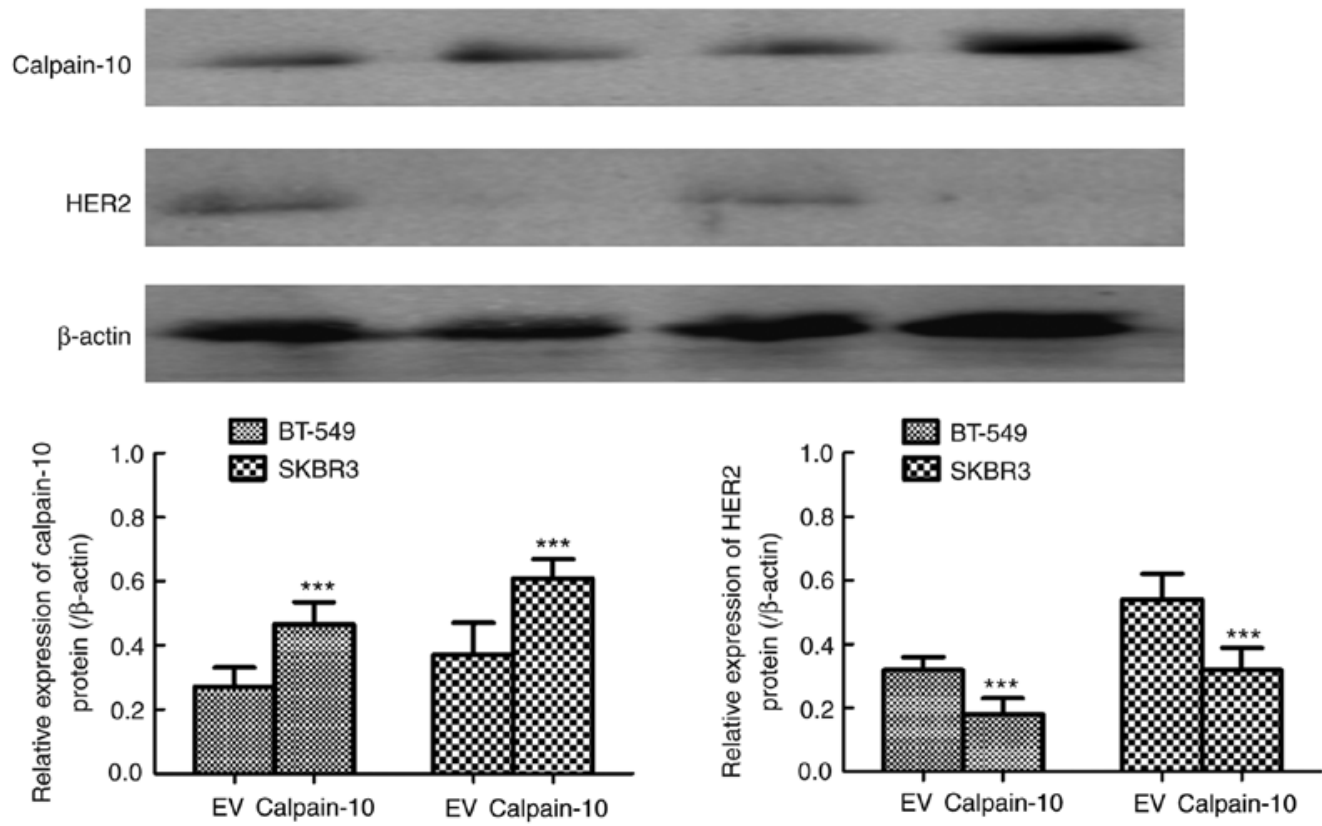

Figure 5. Relative expression of calpain-10 and HER2 in SKBR3 and BT-549 cells after transfection (upregulation of calpain-10) at the mRNA and protein levels. (A) Relative expression of calpain-10 and HER2 in SKBR3 and BT-549 after transfection (upregulation of calpain-10; calpain-10 group) at the mRNA level. (B) Relative expression of calpain-10 and HER2 in SKBR3 and BT-549 after transfection (upregulation of calpain-10) at the protein level. ${ }^{* * *} \mathrm{P}<0.001$, compared with the empty vector (EV) group. HER2, human epidermal growth factor receptor 2.

stage of BC reflects metastatic lymph nodes and a higher histological grade, and the presence of angioma thrombus indicates a higher degree of tumor invasion and a poor prognosis of BC patients (12). Our results showed that clinical stage, lymph node metastasis and histological grade were positively correlated with sHER2-ECD levels $(r=0.320,0.292,0.217$ and 0.285 , respectively). We followed up these 289 patients, and the results showed that the disease-free survival (DFS) and overall survival (OS) of the patients in the sHER2-ECD-negative group were markedly higher compared with those in the sHER2-ECD-positive group, while the cumulative incidence rate (CIR) in the sHER2-ECD-negative group was markedly lower than that of the sHER2-ECD-positive group. Several trials have also demonstrated that SHER2-ECD is a prognostic indicator for BC $(12,13)$. In patients with advanced BC, many studies have suggested that elevated sHER2-ECD level is an independent risk factor for poor prognosis (14-16). Our findings were consistent with other studies. Therefore, we concluded that sHER2-ECD is a prognostic indicator for BC patients, regardless of whether they presented with advanced $\mathrm{BC}$.
We also observed the relationship between sHER2-ECD and Miller and Payne (MP) levels. Among the 36 patients with neoadjuvant chemotherapy, the proportion of sHER2-ECD-positive patients reaching the MP5 level was significantly higher than that of the sHER2-ECD-negative group. However, positive sHER2-ECD was associated with poor prognosis. This may be attributed to the fact that the elevated HER2-ECD in the serum was the ECD of the HER2 molecule, and most of the immunohistochemical (IHC) results of the patients with positive ECD were also positive. Therefore, the patients with positive ECD had more chemotherapeutic drugs to choose from than the ECD-negative patients, such as anti-HER2 therapy, and the target site of anti-HER2 drugs was HER2-ECD (17). Thus, the treatment was more effective, and more patients reached the MP5 level. However, the poor prognosis of patients in the sHER2-ECD-positive group may be attributed to the fact that HER2-ECD was shed into the blood after chemotherapy in some patients, leading to the absence of anti-HER2 treatment targets, drug inefficacy or drug resistance, and the 
Table V. Change in the HER2-ECD level (ng/ml) in culture medium of BT-549 and SKBR3 cell lines before and after downregulation of calpain- 10 .

\begin{tabular}{lccr}
\hline Cell line & Before transfection & After transfection & P-value \\
\hline BT-549 & $19.6 \pm 0.33$ & $10.2 \pm 0.38$ & 0.001 \\
SKBR3 & $24.7 \pm 0.42$ & $11.4 \pm 0.47$ & $<0.001$ \\
\hline
\end{tabular}

HER2-ECD, human epidermal growth factor receptor 2-extracellular domain.

intracellular end of HER2 molecule (HER2-ICD) containing tyrosine kinase domain still existed. This region can still activate the PI3K/Akt signaling pathway, eventually leading to cell transformation, proliferation and resistance to cell death, promoting cell survival, tumor occurrence and development (3).

In addition, we analyzed the association between tissue HER2 status (IHC and/or FISH detection) and sHER2-ECD. Briefly, 29.94\% (50/167) of patients with histologically positive HER2 (IHC 3+ and/or FISH +) had positive sHER2-ECD, while only $12.30 \%(15 / 122)$ of patients with histologically negative HER2 had positive sHER2-ECD, which was consistent with the positive rate reported by others (12). This finding also indicated that SHER2-ECD was correlated with the histological status of HER2, and such a correlation could assist in determining the HER2 status of patients and provide more information for the treatment of patients.

As a ubiquitous non-classical calpain enzyme, calpain-10 belongs to the calpain family. It is a superfamily of intracellular cysteine proteases, which are highly conserved from bacteria to mammals. As a genetic factor, calpain-10 is involved in the development of various human tumors (18). We followed up 289 patients for 23-30 months, and the results revealed that compared with the low-expression group, the overexpression group of calpain-10 had shorter DFS, higher CIR, and worse prognosis. Although there was no statistical difference in OS between the two groups, the OS of the overexpression group of calpain-10 was markedly lower compared with the low-expression group. This may be attributed to the fact that we had a short follow-up time, thus the difference was not statistically significant. Our follow-up results indicated that overexpression of calpain-10 was associated with poor prognosis, which was similar to the results of Storr et al (19) and Chan et al (20). Storr $e t$ al demonstrated that abnormal expression and activation of calpain in BC are associated with poor prognosis (19). IHC evaluation of calpain-10 in patients with esophageal squamous cell carcinoma by Chan et al showed that the high level of calpain-10 was associated with significantly reduced 5-year survival rate (20). Previous studies have shown that the 5-year survival rate of HER2-positive patients is lower compared with HER2-negative patients, indicating that positive HER2 predicts poor prognosis (17). Elevated sHER2-ECD is associated with poor prognosis of BC (16). IHC tests of calpain-10 and HER2 in 289 BC patients showed a positive correlation $(\mathrm{R}=0.178)$. We also found a positive correlation between sHER2-ECD and calpain-10 $(\mathrm{R}=0.439)$. This finding also
Table VI. Change in the HER2-ECD level in culture medium of BT-549 and SKBR3 cell lines before and after upregulation of calpain- 10 .

\begin{tabular}{lccr} 
Cell line & Before transfection & After transfection & P-value \\
\hline BT-549 & $19.6 \pm 0.25$ & $27.5 \pm 0.31$ & $<0.001$ \\
SKBR3 & $24.7 \pm 0.27$ & $31.3 \pm 0.32$ & 0.001 \\
\hline
\end{tabular}

HER2-ECD, human epidermal growth factor receptor 2-extracellular domain.

confirmed the value of calpain-10 in predicting the prognosis of $\mathrm{BC}$ patients.

Calpain-10 plays a crucial role in the uptake of HER2-ECD into the bloodstream (21). To further verify the relationship between SHER2-ECD and calpain-10, we conducted a series of in vitro experiments. We selected two cell lines, BT-549 and SKBR3, from the commonly used BC cell lines, which have high expression of calpain-10 and HER2. First, we downregulated or upregulated calpain-10 expression in both cell lines, and found that the concentrations of HER2-ECD were decreased or increased accordingly in both cell cultures. This finding further confirmed that calpain- 10 plays a fundamental role in ECD shedding into the bloodstream. The detection of HER 2 by IHC method was based on the synthesis of peptide antigens that bind to the ECD of the HER2 molecule, reflecting the ECD level of the HER2 molecule (22). Chemiluminescence was used to detect the concentration of HER2-ECD in serum, and the ECD of the HER2 molecule was also detected. With the decrease or increase of calpain-10, the expression of HER2 was increased or decreased accordingly, showing that there was a negative correlation between calpain-10 and HER2, which also confirmed the shear effect of calpain-10 on ECD. However, the IHC results of 289 clinical samples showed a positive correlation between calpain-10 and HER2, which seemed to be contradictory. We speculate that there is a certain mechanism to initiate or enhance the shear effect of calpain-10 on HER2-ECD, but when this mechanism does not function, Calpain-10 and HER2 are overexpressed. According to the results of the in vivo and in vitro experiments, we believed that calpain-10 and sHER2-ECD play an important role in the treatment and management of $\mathrm{BC}$ patients, which could help more accurately determine the HER2 status of patients. sHER2-ECD levels, calpain-10 and HER2 were detected in $\mathrm{BC}$ patients prior to chemotherapy. If all three indicators are positive, patients could choose anti-HER2 treatment. Due to the positive sHER2-ECD, the binding sites of trastuzumab may be less, and the choice of lapatinib may be more reliable. If tissues are positive for calpain-10 and HER2, and the sHER2-ECD is negative, then trastuzumab could be selected as the first choice. However, when calpain-10 is positive, HER2-ECD may be cut off and shed into the blood, and the sHER2-ECD concentration must be tested regularly. Once the concentration of HER2-ECD is increased, the binding sites of trastuzumab are decreased or even disappear. Lapatinib should be replaced to continue treatment. If tissue calpain-10 and sHER2-ECD are positive and tissue HER2 is 
negative, the FISH test is recommended in order to determine whether HER2 is true-negative or false-negative due to the shear action of calpain-10. If the FISH result is positive, then lapatinib could be selected. If the FISH result is negative, positive sHER2-ECD may be caused by the heterogeneity of tumor tissue, and anti-HER2 treatment should not be selected at this time. If tissue HER 2 is positive and both sHER2-ECD and tissue calpain-10 are negative, anti-HER 2 treatment could be selected without considering the shear effect of calpain-10 and without monitoring sHER2-ECD to reduce treatment cost of patients. If both HER2 in the tissue and HER2-ECD in the serum are negative, anti-HER2 therapy should not be considered regardless of the expression of calpain-10 in the tissue.

Compared with the detection of HER2 level alone, the combined detection of sHER2-ECD, tissue HER2 and calpain-10 could more accurately assess the HER2 level, providing a more comprehensive reference for the treatment of patients. Serum HER2-ECD and tissue calpain-10 levels are powerful factors with which to assess the status of HER2. The combination of these three indicators could enable doctors to more accurately select treatment methods and further realize individualized treatment.

\section{Acknowledgements}

Not applicable.

\section{Funding}

The present study was partially supported by the Health department of China of Hebei province (grant no. 20150772).

\section{Availability of data and materials}

The datasets used and/or analyzed during the current study are available from the corresponding author on reasonable request.

\section{Authors' contributions}

YD, MM and SG designed the present study, summarized and analyzed data, wrote and interpreted the manuscript. QL, SL and JL collected and analyzed the data. CG designed the present study, interpreted the data, and had the final approval of the manuscript. All authors read and approved the manuscript and agree to be accountable for all aspects of the research in ensuring that the accuracy or integrity of any part of the work are appropriately investigated and resolved.

\section{Ethics approval and consent to participate}

This research was approved by the Ethics Committee of the Fourth Hospital of Hebei Medical University and all informed consents were signed by patients.

\section{Patient consent for publication}

Not applicable.

\section{Competing interests}

The authors declare that they have no competing interests.

\section{References}

1. Slamon DJ, Clark GM, Wong SG, Levin WJ, Ullrich A and McGuire WL: Human breast cancer: Correlation of relapse and survival with amplification of the HER-2/neu oncogene. Science 235: 177-182, 1987.

2. Harari D and Yarden Y: Molecular mechanisms underlying ErbB2/HER2 action in breast cancer. Oncogene 19: 6102-6114, 2000.

3. Panis C, Pizzatti L, Corrêa S, Binato R, Lemos GF, da Silva do Amaral Herrera AC, Seixas TF, Cecchini R and Abdelhay E: The positive is inside the negative: HER2-negative tumors can express the HER2 intracellular domain and present a HER2-positive phenotype. Cancer Lett 357: 186-195, 2015.

4. Carney WP, Neumann R, Lipton A, Leitzel K, Ali S and Price CP: Potential clinical utility of serum HER-2/neu oncoprotein concentrations in patients with breast cancer. Clin Chem 49: 1579-1598, 2003.

5. Liu PC, Liu X, Li Y, Covington M, Wynn R, Huber R, Hillman M, Yang G, Ellis D, Marando C, et al: Identification of ADAM10 as a major source of HER2 ectodomain sheddase activity in HER2 overexpressing breast cancer cells. Cancer Biol Ther 5: 657-664, 2006.

6. Rydén L, Landberg G, Stål O, Nordenskjöld B, Fernö M and Bendahl PO: HER2 status in hormone receptor positive premenopausal primary breast cancer adds prognostic, but not tamoxifen treatment predictive, information. Breast Cancer Res Treat 109: 351-357, 2008.

7. Wolff AC, Hammond MEH, Allison KH, Harvey BE, Mangu PB, Bartlett JMS, Bilous M, Ellis IO, Fitzgibbons P, Hanna W, et al: Human epidermal growth factor receptor 2 testing in breast cancer: American society of clinical oncology/college of American pathologists clinical practice guideline focused update. J Clin Oncol 36: 2105-2122, 2018.

8. Livak KJ and Schmittgen TD: Analysis of relative gene expression data using real-time quantitative PCR and the 2(-Delta Delta C(T)) method. Methods 25: 402-408, 2001.

9. Singletary SE, Allred C, Ashley P, Bassett LW, Berry D, Bland KI, Borgen PI, Clark G, Edge SB, Hayes DF, et al: Revision of the American joint committee on cancer staging system for breast cancer. J Clin Oncol 20: 3628-3636, 2002.

10. Ogston KN, Miller ID, Payne S, Hutcheon AW, Sarkar TK, Smith I, Schofield A and Heys SD: A new histological grading system to assess response of breast cancers to primary chemotherapy: Prognostic significance and survival. Breast 12: 320-327, 2003.

11. Qi M, Li JF, Xie YT, Lu AP, Lin BY and Ouyang T: Weekly paclitaxel improved pathologic response of primary chemotherapy compared with standard 3 weeks schedule in primary breast cancer. Breast Cancer Res Treat 123: 197-202, 2010.

12. Ludovini V, Gori S, Colozza M, Pistola L, Rulli E, Floriani I, Pacifico E, Tofanetti FR, Sidoni A, Basurto C, et al: Evaluation of serum HER2 extracellular domain in early breast cancer patients: Correlation with clinicopathological parameters and survival. Ann Oncol 19: 883-890, 2008.

13. Saghatchian M, Guepratte S, Hacene K, Neumann R, Floiras JL and Pichon MF: Serum HER-2 extracellular domain: Relationship with clinicobiological presentation and prognostic value before and after primary treatment in 701 breast cancer patients. Int J Biol Markers 19: 14-22, 2004.

14. Müller V, Witzel I, Lück HJ, Köhler G, von Minckwitz G, Möbus V, Sattler D, Wilczak W, Löning T, Jänicke F, et al: Prognostic and predictive impact of the HER-2/neu extracellular domain (ECD) in the serum of patients treated with chemotherapy for metastatic breast cancer. Breast Cancer Res Treat 86: 9-18, 2004.

15. Fehm T, Jäger W, Krämer S, Sohn C, Solomayer E, Wallwiener D and Gebauer G: Prognostic significance of serum HER2 and CA 15-3 at the time of diagnosis of metastatic breast cancer. Anticancer Res 24: 1987-1992, 2004.

16. Wang T, Zhou J, Zhang S, Bian L, Hu H, Xu C, Hao X, Liu B, Ye Q, Liu Y and Jiang Z: Meaningful interpretation of serum HER2 ECD levels requires clear patient clinical background, and serves several functions in the efficient management of breast cancer patients. Clin Chim Acta 458: 23-29, 2016.

17. Pohlmann PR, Mayer IA and Mernaugh R: Resistance to trastuzumab in breast cancer. Clin Cancer Res 15: 7479-7491, 2009.

18. Moretti D, Del Bello B, Allavena G and Maellaro E: Calpains and cancer: Friends or enemies? Arch Biochem Biophys 564: 26-36, 2014. 
19. Storr SJ, Lee KW, Woolston CM, Safuan S, Green AR, Macmillan RD, Benhasouna A, Parr T, Ellis IO and Martin SG: Calpain system protein expression in basal-like and triple-negative invasive breast cancer. Ann Oncol 23: 2289-2296, 2012.

20. Chan D, Tsoi MY, Liu CD, Chan SH, Law SY, Chan KW, Chan YP, Gopalan V, Lam AK and Tang JC: Oncogene GAEC1 regulates CAPN10 expression which predicts survival in esophageal squamous cell carcinoma. World J Gastroenterol 19: 2772-2780, 2013.
21. Leloup L and Wells A: Calpains as potential anti-cancer targets. Expert Opin Ther Targets 15: 309-323, 2011.

22. Carney WP, Hamer PJ, Petit D, Retos C, Greene R, Zabrecky JR, Mc Kenzie S, Hayes D, Kufe D, DeLellis R, et al: Detection and quantitation of the human neu protein. J Tumor Marker Oncol 6: 53-72, 1991. 